\title{
ESTIMACIÓN DEL ESFUERZO EFECTIVO PARA SUPERAR LA ASIGNATURA DE ESTADÍSTICA DESCRIPTIVA EN LAS LICENCIATURAS DE ECONOMÍA Y ADMINISTRACIÓN DE EMPRESA \\ [Student workload estimation to pass a statistics course in Economics and Business Administration]
}

por

$\underline{\text { Article record }}$

$\underline{\text { About authors }}$

HTML format $\underline{\text { Ficha del artículo }}$

$\underline{\text { Sobre los autores }}$

Formato HTML

\author{
Jano, María Dolores (dolores.jano@uam.es) \\ Ortiz Serrano, Salvador (salvador.ortiz@uam.es)
}

\section{Resumen}

El proceso de convergencia en la Educación Superior en Europa implica un cambio radical en la enseñanza superior. Una de las cuestiones fundamentes de la armonización radica en el proceso de asignación de créditos ECTS (European Credit Transfer System). En este artículo se analizan diversas metodologías para la estimación de la carga de trabajo del estudiante y se presentan algunos resultados en relación al tiempo de estudio para superar la asignatura de Estadística Descriptiva en las licenciaturas de Economía y ADE. La estimación obtenida está ligeramente por debajo de las 25-30 horas por crédito que usualmente se establecen como referencia.

\section{Descriptores}

Recogida y análisis de datos, aprendizaje activo, ECTS, Esfuerzo Efectivo del estudiante.

Madrid. El objetivo general del proyecto fue realizar una experiencia en innovación educativa para poner en marcha iniciativas de mejora de la calidad en el ámbito de la docencia en estadística económica ante el reto que supone la implantación definitiva antes del año 2010 del Espacio Europeo de Educación Superior. Este nuevo sistema pone el 
Jano, M.D. y Ortiz Serrano, S. (2007). Estimación del esfuerzo efectivo para superar la asignatura de estadística descriptiva en las Licenciaturas de Economía y Administración de Empresas.

RELIEVE, v. 13, n. 2, p. 191-202. http://www.uv.es/RELIEVE/v13n2/RELIEVEv13n2_3.htm

énfasis en el aprendizaje del estudiante, en la calidad de la enseñanza, y proporciona una magnífica oportunidad para transformar paradigmas de enseñanza y aprendizaje. Uno de los aspectos claves de la reforma es el establecimiento de un sistema de créditos ECTS (European Credit Transfer System) basados en el esfuerzo efectivo o carga de trabajo que un estudiante medio requiere para superar la asignatura, frente al modelo tradicional que tiene en cuenta exclusivamente el número de horas de clase.

En este artículo se ofrece una estimación del tiempo que un estudiante medio requiere para aprobar la asignatura de Estadística Descriptiva en las licenciaturas de Economía y Administración de Empresas. En primer lugar, se analiza el contexto o marco en el que surge la iniciativa de innovación y los diversos procedimientos existentes para medir el esfuerzo efectivo. Seguidamente, se describe el método utilizado en la recogida y depuración de los datos. A continuación, se ofrecen los resultados obtenidos por un grupo de 6 profesores del Departamento de Economía Aplicada que impartieron docencia en la asignatura troncal de Estadística Descriptiva, en la que estaban matriculados algo más de 650 estudiantes. Por último, se resumen las conclusiones más relevantes y algunas implicaciones para el proceso de enseñanza aprendizaje que se desprenden de la experiencia realizada.

\section{2.- Marco conceptual}

Existe abundante información disponible sobre el Espacio Europeo de Educación Superior. ${ }^{[1]}$ En síntesis, se puede decir que el proceso de convergencia arranca en la Declaración de Bolonia en junio de 1999 que establece las bases para la construcción de un "Espacio Europeo de Educación Superior". El proceso de armonización se ha ido configurando posteriormente con una serie de comunicados y declaraciones: Praga 2001, Berlín 2003 y Bergen 2005.
En España, el Real Decreto 1125/2003 fija en sus artículos 3 y 4 el concepto de crédito ECTS y la asignación de los mismos. El Real Decreto establece que: "En la asignación de créditos a cada una de las materias que configuren el plan de estudios se computará el número de horas de trabajo requeridas para la adquisición por los estudiantes de los conocimientos, capacidades y destrezas correspondientes. En esta asignación deberán estar comprendidas las horas correspondientes a las clases lectivas, teóricas o prácticas, las horas de estudio, las dedicadas a la realización de seminarios, trabajos, prácticas o proyectos, y las exigidas para la preparación y realización de los exámenes y pruebas de evaluación. Esta asignación de créditos, y la estimación de su correspondiente número de horas, se entenderá referida a un estudiante dedicado a cursar a tiempo completo estudios universitarios durante un mínimo de 36 y un máximo de 40 semanas por curso académico. El número mínimo de horas, por crédito, será de 25, y el número máximo, de 30".

La carga de trabajo de un estudiante medio es un concepto muy difícil de definir, y en ocasiones es visto con recelo por los profesores como señala Lavigne (2003). Para algunos profesores no es mesurable, máxime si la única forma de estimarlo es preguntar a los estudiantes, que se puede suponer que no van a dar respuestas fidedignas. Otros señalan que lo que importa no es cuántas horas trabaja un estudiante, sino cuánto aprende en un tiempo dado, ya que el tiempo que van a dedicar a una asignatura depende de factores como la cantidad de tiempo que disponen según el número de asignaturas matriculadas y las tareas que se proponen en ellas. Además, otro argumento por el que muchos académicos manifiestan una oposición frontal a este sistema, es que consideran que el tiempo que necesitan depende de los talentos, de los niveles o conocimientos previos de los estudiantes y de la dificultad o grado de ansiedad que provoca la asignatura, por lo que resulta imposible determinar el tiempo que le llevará 
a un estudiante medio cumplir con el trabajo requerido.

Existen diversos procedimientos para determinar la carga de trabajo del estudiante. A continuación, a modo de resumen, no exhaustivo, se presentan algunos de ellos.

- Realizar encuestas a los estudiantes durante el proceso de aprendizaje, tanto semanalmente como retrospectivas al finalizar el curso. La mayoría de Universidades Españolas ha realizado encuestas de este tipo.

- Pedir a los estudiantes que mantengan un diario en el que apunten el tipo de actividad, el tiempo de estudio, y la productividad, como propone Valero-García (2003).

- Que los profesores realicen una planificación de los contenidos y actividades de aprendizaje con estimación del tiempo requerido para cada actividad. El proyecto Tuning ofrece modelos y ejemplos de planificación de módulos o asignaturas para ser cumplimentadas por el profesor y por los estudiantes [2].

- Realizar encuestas retrospectivas a los profesores al finalizar el periodo de estudio sobre cuánto tiempo creen que necesita el estudiante medio para conseguir los objetivos de aprendizaje ${ }^{[3]}$.

- Determinación de una equivalencia entre horas de clase de teoría ó práctica y el tiempo de estudio requerido, fijando un coeficiente de ajuste o un cambio de escala sobre las horas de clase en el aula [4].

- Llevar un diario en el que los estudiantes anotan cada hora las actividades que realizan a lo largo de una semana en intervalos horarios desde las 8 de la mañana hasta las 12 de la noche. En el diario se recoge no sólo información cuantitativa, sino también cualitativa para aclarar y precisar lo que sea necesario las actividades que se realizan. Esta información se complementa con una serie de entrevistas en profundidad a una submuestra, que permiten realizar inferencias a través de un estudio detallado de esos casos. (Kember, 2004).
- Las universidades a distancia suelen hacer estimaciones de tiempo que requiere un estudiante para procesar y comprender diversos materiales de estudio. En base a esos estudios se suele determinar aspectos como tiempo requerido para leer una página, para escribir una página de investigación sobre un tema, para preparar exámenes, etc. No obstante, estas tasas son muy dependientes de factores como grado de dificultad del material y del campo o disciplina en que se desarrolla la docencia. (Chamber, 1992).

En este proyecto se ha optado por preguntar a los estudiantes el tiempo que semanalmente dedican a estudiar. También se solicita información sobre una serie de características para analizar las relaciones entre esfuerzo efectivo y una serie variables explicativas del mismo. Además, para verificar si hay ajuste entre lo que los estudiantes trabajan y lo que se espera de ellos, se ha contrastado la opinión de los estudiantes con lo que los profesores estimaron que deberían estudiar.

\section{3.- Metodología}

La metodología utilizada a lo largo del curso, así como algunos resultados de la experiencia de innovación docente pueden consultarse en Jano, M.D. y Ortiz S. (2007). En la estimación del tiempo que un estudiante medio requiere para superar la asignatura se han considerado dos procedimientos alternativos:

- Preguntar directamente a los estudiantes.

- Preguntar a los profesores responsables de la asignatura que han participado en el proyecto.

\section{1.- Estimación del tiempo de estudio a partir de la información que propor- cionan los estudiantes}

El colectivo objeto de estudio han sido los estudiantes matriculados en el curso 2004/05 en la asignatura de estadística descriptiva, en los 5 grupos asignados a los 6 profesores implicados en el proyecto, un total de 410 en 
la licenciatura en Economía y 246 en la Licenciatura de Administración y Dirección de Empresas. De esos 656 estudiantes, algo menos de la mitad eran repetidores. Inicialmente, se contempló la posibilidad de preguntar a un panel de estudiantes seleccionados al azar y efectuar un seguimiento de los mismos en relación al tiempo de estudio y el grado de dificultad de los distintos temas según se iban explicando. Sin embargo, diversas entrevistas con estudiantes de cursos pasados y la propia experiencia docente, pusieron de manifiesto algunas dificultades que se podrían encontrar en el proceso de recogida de los datos:

- Localizar a los estudiantes que no asisten a clase de manera regular para solicitar su colaboración resulta una tarea complicada y costosa.

- Reticencia de los alumnos a informar a sus profesores sobre el tiempo que dedican a estudiar, sobre todo si creen que se les va a controlar o si su estrategia es estudiar sólo los últimos días antes del examen.

- Conveniencia de un registro del tiempo de estudio de periodicidad al menos semanal, porque periodos más largos entrañan riesgo de olvido o errores, bien por subestimación o por sobreestimación del tiempo efectivamente dedicado a estudiar.

- Alta tasa de abandonos previsible, puesto que la colaboración de un estudiante las primeras semanas, no garantiza que continúe haciéndolo las semanas restantes.

- Dificultad de pronosticar al principio del curso qué estudiantes van a superar la asignatura, de modo que, dado que el objetivo es estimar el tiempo que necesita un estudiante medio para aprobar la asignatura, habrá que recoger información de todos los estudiantes.

La consideración de los problemas anteriormente mencionados aconsejó adoptar una estrategia más práctica, aunque quizá con algunos sesgos, consistente en realizar semanalmente una encuesta a los alumnos que asistían a clase, poniendo también a su disposición los cuestionarios en la página web de la asignatura. En general, la colaboración a lo largo del curso fue aceptable, aunque el hecho de que el alumno tuviera que anotar su DNI al realizar las encuestas, podría conllevar errores de medida en los datos. Cabe esperar que los estudiantes, al ser preguntados por sus profesores sobre el tiempo que dedican a estudiar, opten por no responder o por declarar que estudian más de lo que realmente lo hacen. Sin embargo, dado que el interés se centra en estimar el esfuerzo que se requiere para aprobar la asignatura, se hace indispensable poder asociar tiempo de estudio con calificación obtenida, por lo que las encuestas no podían ser anónimas.

La información se recogió a lo largo de 17 semanas. En el cuestionario, muy breve, se les hacían las siguientes preguntas:

"Sin contar las horas de asistencia a clase ¿Cuánto tiempo has dedicado esta semana a preparar todas las asignaturas en las que estas matriculado? (Incluye pasar apuntes, estudiar teoría, hacer ejercicios, consultar bibliografía, asistir a tutorías, consultar en Internet, etc. ) (Horas, minutos)

De ese tiempo ¿Cuánto has dedicado a preparar Estadística Descriptiva?. (Horas, minutos)

$Y$ del tiempo en Estadística Descriptiva, ¿Qué porcentaje has dedicado a resolver ejercicios? (cualitativa en 5 intervalos)”.

La correcta interpretación de los resultados requiere señalar algunas situaciones especiales e incidencias observadas a lo largo del proceso de recogida:

- Los estudiantes que aprobaron la selectividad en septiembre, sobre todo en economía, se incorporaron al grupo unas semanas más tarde que el resto. Sin embargo, dado que se observa que el comportamiento de estudio en las primeras semanas de estudios es bastante similar, se estimó que la primera semana de curso, en la que se presentó la asignatura y la metodología docente, el tiempo de estudio sería míni- 
mo. Además, se supone como hipótesis de trabajo que en las primeras semanas se estudia más o menos lo mismo, de manera que se estiman la segunda y tercera semana del curso con el mismo tiempo que el dedicado a la cuarta semana.

- Todos los grupos pertenecientes al proyecto tenían dos horas de clase los martes, en este cuatrimestre hubo 3 fiestas en martes, por lo que se perdieron varias horas de clase. Esto afecto tanto al número total de horas efectivas de clase a lo largo del curso, como al proceso de recogida de la información.

- En el periodo de vacaciones de navidad, casi tres semanas, hubo que recoger los datos al regreso de las mismas. La tasa de respuesta fue ligeramente más baja, y cabe esperar una peor calidad de los datos, puesto que no todos los alumnos registraron semanalmente los datos y dieron una aproximación del tiempo de estudio en base a su recuerdo.

- El sistema de evaluación continua consistía en la realización de dos controles a lo largo del curso, y un examen global de toda la asignatura, y se observa un comportamiento estacional en el tiempo de estudio en estadística, de manera que, a medida que se aproxima la fecha de los exámenes se observan mayores valores de tiempo de estudio.

- Los cuestionarios referidos al periodo de exámenes se entregaron en clase y se pusieron a disposición de los estudiantes en la web de la asignatura. Los alumnos podían entregarlo en cualquier momento en el despacho del profesor y además se pasó por las clases la segunda semana del segundo semestre con cuestionarios adicionales. Se observó una menor tasa de participación y una peor calidad de la información recogida, puesto que la mayoría de los estudiantes no registraron semana tras semana el tiempo de estudio, sino que ofrecieron una estimación en base al recuerdo de todo el periodo, y la tasa de respuesta fue muy baja. Afortunadamente, para aquellos estudiantes que aprobaron la asignatura y no se presentaron a subir nota al examen final, el tiempo dedicado a estudiar la asignatura se supone que es nulo y no perturba demasiado la estimación del tiempo total. No ocurre lo mismo con la estimación del tiempo total dedicado al resto de asignaturas, o para los estudiantes que no han superado la asignatura por evaluación continua.

- Aunque se pusieron todos los medios para facilitar y promover la participación de los estudiantes (carta inicial explicando la importancia de los datos que se iban a recoger y el compromiso de tratarlos sin identificar al estudiante, insistir al principio y al final de clase, insistir el resto de días de clase, encuestas retrospectivas para las semanas que no habían colaborado, cuestionarios en la web que se podían entregar en cualquier momento en el despacho del profesor, incluso se ofreció la posibilidad de enviar la encuesta por correo electrónico), se observa una disminución progresiva de la tasa de participación (desde un 55\% del total de alumnos matriculados en la prueba de nivel realizada en clase a un $20 \%$ en la última recogida de información). Esto puede ser debido a los abandonos de aquellos que deciden dejar la asignatura para septiembre, y al agotamiento que supone para los estudiantes que siguen preparando la asignatura rellenar semanalmente la encuesta.

Se realizó un proceso de depuración de los datos obtenidos, descartando aquellos que ofrecían valores extraordinariamente más altos de lo razonable o incongruentes, confiando en general en la honestidad y veracidad de sus respuestas obtenidas. Por tanto, los resultados que se ofrecen a continuación son el producto de la información que los estudiantes han proporcionado, con sus ventajas y sus limitaciones.

A la vista de estos resultados, y dado que el objetivo es estimar el tiempo de estudio para superar la asignatura, se decide centrar la atención en el colectivo de estudiantes que 
Jano, M.D. y Ortiz Serrano, S. (2007). Estimación del esfuerzo efectivo para superar la asignatura de estadística descriptiva en las Licenciaturas de Economía y Administración de Empresas.

RELIEVE, v. 13, n. 2, p. 191-202. http://www.uv.es/RELIEVE/v13n2/RELIEVEv13n2_3.htm

aprobaron. A su vez, entre ellos se diferencian tres colectivos:

- Los que no han respondido a ninguna encuesta.

- Los que han respondido a menos de 8 semanas de las 17 posibles.

- Los que han respondido a 8 y más semanas.

Se consideró que la información más fiable, aunque seguramente algo sesgada, era la que proporcionaron los estudiantes que responden al menos 8 semanas, y se decidió utilizar algún procedimiento de imputación para obtener la información que falta ${ }^{[5]}$. Así pues, finalmente se estimó el esfuerzo efectivo para superar la asignatura, y los factores de los que depende con un total de 153 estudiantes.

Además estos resultados se complementan con la información proporcionada por los estudiantes que han colaborado menos de 8 semanas y con los datos obtenidos a través de una breve encuesta telefónica a aquellos que no rellenaron ninguna ficha. En la Tabla 1 se presentan algunos datos sobre la colaboración de los estudiantes en el proceso de recogida de datos.

Tabla 1.- Distribución de los alumnos que aprueban la asignatura según su colaboración en el proyecto

\begin{tabular}{|l|c|}
\cline { 2 - 2 } \multicolumn{1}{c|}{} & $\mathrm{N}^{\mathrm{o}}$ de alumnos \\
\hline Responden 8 ó mas semanas & 153 \\
Encuesta telefónica & 39 \\
No se dispone de información & 12 \\
\hline Total de aprobados & 42 \\
\hline
\end{tabular}

\section{2.- Procedimiento usado en la esti- mación del tiempo de estudio a partir de la información que proporcionan los profesores responsables de la asignatura que han participado en el proyecto}

El procedimiento seguido consistió en invitar a los profesores participantes en el proyecto de innovación a realizar una reflexión a lo largo del curso sobre su práctica docente y sobre el tiempo que consideraban que debía dedicar un estudiante medio a preparar la asignatura. La información se recogió de manera cualitativa en las diversas reuniones de coordinación realizadas a lo largo del curso, y de manera formal en una reunión final de valoración del proyecto realizado.

Se crearon una serie de documentos para promover la reflexión y homogeneizar los criterios a utilizar en la estimación del tiempo de estudio:
- Actas de las reuniones celebradas, con un resumen de los temas tratados y los acuerdos alcanzados.

- Ficha mensual de seguimiento del Proyecto de Innovación docente, en la que se recogía información sobre asistencia y participación de los estudiantes en clase, y además una valoración del tiempo estimado para la realización por los estudiantes de las tareas propuestas.

- Encuesta final de evaluación de la experiencia de innovación docente, en la que se solicitaba una valoración del proceso de enseñanza-aprendizaje propuesto, y sobre la carga adicional de trabajo que supuso para los profesores participantes en ella.

\section{4.- Resultados}

Los resultados se analizan desde la doble perspectiva de la información que proporcionan estudiantes y profesores. 


\section{1.- Según los datos proporcionados por estudiantes}

La información proporcionada por los estudiantes nos permite concluir que en promedio se requieren 63 horas para preparar la asignatura de Estadística Descriptiva. No obstante, se observa una gran dispersión en la estimación del tiempo medio de estudio, entre un tiempo mínimo de 20 horas y uno máximo de 143 horas. Además el 25\% de estudiantes que menos estudia dice que necesita como mucho 44,5 horas, el $50 \%$ que menos estudian dedica menos de 59 horas y el $25 \%$ de los más estudiosos estudia al menos 78 horas. ${ }^{[6]}$ Hay que destacar que en esta estimación no se ha tenido en cuenta el tiempo que ya dedicaron en cursos anteriores a preparar la asignatura los alumnos repetidores.

Para la determinación de los créditos ECTS habría que añadir el tiempo que dedica a otras actividades como asistencia a clase, realización de trabajos, tutorías, exámenes, etc. En este sentido el número total de horas de clase que se impartió fue de 50 horas (se perdieron 10 horas de clase por las fiestas y por la realización de exámenes en las horas de clase). A estas horas lectivas, hay que añadir el tiempo empleado en la realización de exámenes. En este caso se añaden cinco horas (una hora y media por cada control y dos horas para el examen final). Según estos cálculos el esfuerzo efectivo del estudiante ronda las 118 horas como media para este curso, que siguiendo la relación de entre 25 a 30 horas por crédito fijada en el Real Decreto, supondría entre 3,9 y 4,7 créditos ECTS. Suponiendo la relación entre horas lectivas y horas de estudio estable, si realmente se hubieran impartido 60 horas lectivas, las horas de estudio asociadas serían unas 75,6. Teniendo en cuenta además las 5 horas dedicadas a exámenes, se obtendría un esfuerzo efectivo de 140,6 horas como media para la asignatura lo que equivale a entre 4,7 y 5,6 créditos ECTS.

Un análisis de la relación entre el tiempo de estudio en descriptiva observado y algunos factores explicativos nos lleva a la conclusión de que estudian más las mujeres, los que provienen de bachillerato de tecnología, los que cursan $\mathrm{ADE}$, los que aprueban por evaluación continua, y los que obtienen mejores notas. Estos resultados se pueden observar en la Tabla 2.

Esta información se complementa con la información que proporcionan aquellos estudiantes que colaboraron en menos de 8 semanas, y por tanto no permite dar una estimación individualizada para cada uno de ellos del tiempo total. Según estos datos, los estudiantes dedican una media de 185 horas a estudiar, de las cuales 60 horas estudian estadística. En ambos casos, está algo por debajo del tiempo estimado con los alumnos para los que se dispone de más de 8 semanas. 
Jano, M.D. y Ortiz Serrano, S. (2007). Estimación del esfuerzo efectivo para superar la asignatura de estadística descriptiva en las Licenciaturas de Economía y Administración de Empresas.

RELIEVE, v. 13, n. 2, p. 191-202. http://www.uv.es/RELIEVE/v13n2/RELIEVEv13n2_3.htm

Tabla 2. Tiempo medio de estudio empleado en la asignatura de Estadística Descriptiva según características del estudiante

\begin{tabular}{|l|c|c|}
\cline { 2 - 3 } \multicolumn{1}{l|}{} & \% de alumnos & No total de horas \\
\hline Sexo & $39 \%$ & 58,7 \\
MOMBRER & $61 \%$ & 65,5 \\
\hline Opción Bachillerato & $15 \%$ & \\
\hline CIENCIAS Y SALUD & $63 \%$ & 57,3 \\
HUMANIDADES & $22 \%$ & 62,3 \\
TECNOLOGIA & & 65,5 \\
\hline Licenciatura & $51 \%$ & \\
ECONOMIA & $49 \%$ & 59,8 \\
LADE & & 66,3 \\
\hline Evaluación & $87 \%$ & 63,8 \\
\hline APRUEBA POR EVAL. CONT. & $13 \%$ & 57,7 \\
APRUEBA NO EVAL. CONT. & & \\
\hline Calificación final & $44 \%$ & 57,4 \\
\hline APROBADO & $36 \%$ & 65,7 \\
NOTABLE & $20 \%$ & 70,4 \\
\hline SOBRESALIENTE & & $\mathbf{6 2 , 8}$ \\
\hline TOTAL & & \\
\hline
\end{tabular}

Entre los estudiantes que aprobaron, pero que no habían contestado a ninguna de las encuestas realizadas a lo largo del curso se decidió hacer una entrevista telefónica, en la que se le preguntó cuánto tiempo había dedicado como media semanalmente a preparar todas las asignaturas en general, y en particular la estadística descriptiva en los meses de octubre, noviembre, diciembre y enero. Los resultados no difieren mucho de los obtenidos anteriormente, una media aproximada de 62 horas de estudio para superar la asignatura de estadística descriptiva. Sin embargo se observa una gran variabilidad, y por tanto ese promedio tiene una escasa representatividad. De hecho, el 25\% de los estudiantes que menos estudian dicen que estudian como mucho 20 horas, mientras que el $25 \%$ de los que más estudian lo hacen al menos 86 horas. Además, sólo se consiguió información de los 12 estudiantes que quisieron colaborar después de repetidos intentos, lo que supone un $22 \%$ de los posibles ${ }^{[7]}$. Así pues, los resultados han de ser analizados con toda cautela.

Aun teniendo en cuenta las limitaciones que impone el reducido número de estudiantes entrevistados, si se pueden extraer algunas conclusiones:

- Los estudiantes, al ser preguntados sobre las medias semanales en cada mes tenían serias dificultades para ofrecer una estimación en base al recuerdo y por tanto se observa una gran dispersión en los datos, lo que hace que la media de 62 horas sea poco representativa.

- Esto último confirma la necesidad de recoger los datos semanalmente, o al menos obtener el compromiso por parte de los estudiantes de llevar un registro semanal de las horas de estudio para poder dar una medida fiable del tiempo total de estudio que se requiere para superar la asignatura. 


\section{2.- Según las estimaciones realizadas por los profesores}

En la estimación del esfuerzo efectivo que en opinión de los profesores deberían realizar un estudiante medio para superar la asignatura, hay que señalar que no se trata de una tarea sencilla. Además de la falta de hábito en esta tarea, los profesores señalan que cada curso es diferente y cada grupo tiene sus peculiaridades, por lo que habría que atender a estos aspectos. Además, depende mucho de las características del grupo y de los conocimientos previos de los estudiantes. No obstante, y con todos los matices oportunos, en general consideran que el alumno debería dedicar entre 1,5 y 2 veces más de tiempo que el que dedicado a clases en el aula, y entre 15 y 25 horas adicionales para preparar la evaluación final. Así pues, si suponemos una asignatura de 6 créditos, es decir 60 horas lectivas (10 horas por crédito), la estimación estaría entre 165 y 205 horas totales. Teniendo en cuenta que las horas lectivas efectivas fueron 50, la estimación de los profesores se encontraría en un intervalo de 140 a 175 horas.

\section{5.- Discusión}

El objetivo de este trabajo es ofrecer una estimación del tiempo que requiere un estudiante medio para superar la asignatura de Estadística Descriptiva en las licenciaturas de Economía y Administración de Empresas, según el nuevo sistema de créditos ECTS. Para ello, se preguntó a los estudiantes sobre el esfuerzo efectivo que hicieron para superar la asignatura, y a los profesores sobre el que consideraban que se debería realizar.

En el proceso de estimación surgen una serie de dificultades y limitaciones que habrá que tener presentes en la interpretación de los resultados obtenidos, y que se pueden resumir en 3 factores:

a) El cambio de enfoque hacia el aprendizaje activo del estudiante, requiere una respuesta por parte de profesores y estu- diantes, que tendrán que ir modificando progresivamente las actitudes y la manera en que se enfrentan al proceso de enseñanza-aprendizaje.

b) La poca predisposición a colaborar entre profesores y estudiantes para dedicar tiempo a medir el esfuerzo requerido para superar la asignatura, se explica por la falta de incentivos.

c) Los estudiantes, presionados por el miedo a ser controlados por el profesor, o a que tenga consecuencias en la calificación final obtenida, suelen falsear la información, señalando que estudian más de lo que realmente lo hacen.

Los resultados obtenidos permiten señalar que para preparar esta asignatura, de 6 créditos LOU (60 horas lectivas), un estudiante emplea, en media, unas 63 horas, aunque la distribución del tiempo de estudio es bastante heterogénea, observando valores entre 20 y 143 horas. Además, teniendo en cuenta las horas de clase y de realización de exámenes, el tiempo de estudio estimado si se hubieran impartido las 60 horas lectivas, y que un crédito ECTS supone entre 25 y 30 horas de esfuerzo del estudiante, se obtiene una estimación de entre 4,7 y 5,6 créditos ECTS para la asignatura de Estadística Descriptiva.

La experiencia llevada a cabo suscita algunas consideraciones:

El esfuerzo efectivo del estudiante dependerá mucho del esfuerzo efectivo que el profesor realiza (planificación de la asignatura, metodología docente, sistemas de evaluación); pero también de las características personales del propio estudiante (sus conocimientos previos, su interés en la asignatura, el grado de dificultad que perciba, etc.). Así, un estudiante a priori "pasivo" puede responder muy activamente ante propuestas que le motivan y le presentan el aprendizaje como algo valioso y enriquecedor.

El tiempo y esfuerzo del profesor, normalmente demasiado presionado por el elevado número de estudiantes o por las exigen- 
Jano, M.D. y Ortiz Serrano, S. (2007). Estimación del esfuerzo efectivo para superar la asignatura de estadística descriptiva en las Licenciaturas de Economía y Administración de Empresas.

RELIEVE, v. 13, n. 2, p. 191-202. http://www.uv.es/RELIEVE/v13n2/RELIEVEv13n2_3.htm

cias de obtener méritos en investigación, es menor cuando se trabaja en equipo y todos cooperan para mejorar la calidad.

Los resultados obtenidos, en cuanto a estimación del esfuerzo que debe realizar el estudiante, constituyen un punto de partida que se deberá revisar periódicamente a la luz de los contenidos, habilidades, destrezas y competencias que nuestros estudiantes deban conseguir.

\section{Bibliografía}

ANECA (2004) Programa de Convergencia Europea. El crédito europeo. http://www.aneca.es/publicaciones/docs/pu bli_credito\%20europeo.pdf. Consultado 15 abril de 2005.

Barbosa, J., Guiteras, J. y Fonrodona, G. (2004). Teaching chemistry in Europe -a look at future developments. Anal Bioanal Chem, vol. (378), 33-36.

BOE 18-9-03: REAL DECRETO 1125/2003, de 5 de septiembre, por el que se establece el sistema europeo de créditos y el sistema de calificaciones en las titulaciones universitarias de carácter oficial y validez en todo el territorio nacional. http://www.mec.es/univ/html/informes/EEE S_2003/RD_Creditos_Sistema_calificacion es.pdf. Consultado 22-4-06.

Chambers, E. (1992). Work-load and the quality of student learning. Studies in Higher Education, Jun 92, Vol. 17, Issue 2 , 141-153.

Jano, M.D. y Ortiz, S. (2007). Experiencia de innovación docente en estadística económica. Red U. Revista de Docencia Universitaria, numero 2. Consultado 10/Sept/2007 en http://www.redu.um.es/Red_U/2/

Kember, D. (2004). Interpreting student workload and the factors which shape students' perception on their workload. Studies in Higher Education, Vol. 29, $\mathrm{n}^{0}$ 2, April 2004.

Lavigne, R. de (Marzo 2003). Créditos ECTS y métodos para su asignación. Consultado 22-4-06 http://www.aneca.es/modal_eval/docs/doc_ conv_gral1.pdf

Pagani, R. (2002). Convergencia de Programas al Crédito Europeo (ECTS). Jornadas de docencia. Facultad de Medicina UCM, 25-5-02. [01Pagani2001ConvergdeProgr al CrédEur.doc]. Consultado 22-4-06 en http://www.med.ub.es/ aprats/sae/docencia/ creditosECTS.ppt

Proyecto

Tuning. www.relint.deusto.es/TuningProject/index.h tm . Consultado 22-4-06

Valero García, M. (2003). ¿Cómo nos ayuda el Tour de Francia en el diseño de programas docentes centrados en el aprendizaje?. Versión escrita de la conferencia de clausura de las Jornadas sobre Enseñanza Universitaria de la Informática (Junio 2003), titulada: ¿Qué tienen que ver los créditos ECTS con el Tour de Francia?. [Univ. Politécnica Cataluña]. Consultado 22-4-06 en http://www.eees.ua.es/conferencias/articulo ECTS_Politecnica.pdf

Villar, L.M. (2004). Programa para la mejora de la docencia universitaria. Madrid. Prentice Hall.

Villar, L.M. y Alegre, O. (2004). Manual para la excelencia en la educación superior. Madrid. Mc Graw Hill.

\section{NOTAS}

${ }^{[1]}$ Un excelente resumen de enlaces y principales documentos sobre EEES es la página mantenida por la Facultad de Psicología de la UAM. http://www.um.es/facpsi/Europa/ [2]

http://www.relint.deusto.es/TuningProject/i ndex.htm

${ }^{[3]}$ Por ejemplo, los informes de autoevaluación de la actividad docentes en la UAM.

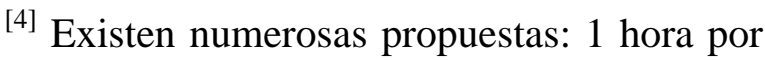
1 hora de teoría, y 2 horas para clases de problemas (Valero García, 2003). Pagani (2002), señalaba de 1,5 a 2 horas por cada hora de teoría y de 0,5 a 1,5 horas por hora de práctica aunque las relaciones dependen del campo científico en que se desarrolle la docencia. Barbosa, J. (2004) en el currículo 
Jano, M.D. y Ortiz Serrano, S. (2007). Estimación del esfuerzo efectivo para superar la asignatura de estadística descriptiva en las Licenciaturas de Economía y Administración de Empresas.

RELIEVE, v. 13, n. 2, p. 191-202. http://www.uv.es/RELIEVE/v13n2/RELIEVEv13n2_3.htm

de química, establece 1 hora de clase requiere 1,5 de trabajo, 1 hora de laboratorio, 1 hora de trabajo adicional.

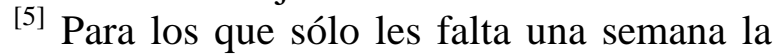
imputación se realiza a través de la media en dicha semana, calculada a partir de los alumnos que tienen su misma nota. El resto de imputaciones se calcula teniendo en cuenta la nota y las proporciones de tiempo que dedican a estudiar cada semana, calculadas a partir de los estudiantes que responden a todas las semanas, puesto que se observó un comportamiento estacional en el estudio, con tiempos más altos cuando se acercaban los exámenes.

[6] Kember (2004, pp. 166) aprecia también una gran variabilidad en la estimación de tiempo de asistencia a clase y de estudio tanto entre semanas diferentes como entre estudiantes.

[7] En general son estudiantes que no han asistido a clase (83,3\% han asistido como mucho a la mitad de la clase), muchos van a una academia (42\%), no han participado en el procedimiento de evaluación continua, la mayoría son de económicas (83,3\%), no es la primera vez que cursan la asignatura (92\% de ellos repiten), y están matriculados en más de 6 asignaturas (42\%).

\section{NOTAS COMPLEMENTARIAS}

[1] Además de los dos autores, participaron en el PID los profesores Begoña Varela, Ana Isabel González, Rocío Marco y Francisco Soto

\section{ABOUT THE AUTHORS / SOBRE LOS AUTORES}

Jano, María Dolores (dolores.jano@uam.es). Es la autora de contacto para este artículo. Profesora Titular del Departamento de Economía Aplicada. Facultad de Ciencias Económicas y Empresariales. Universidad Autónoma de Madrid. C/ Francisco Tomás y Valiente, 5. 28049 - Madrid.

Buscar otros artículos de esta autora en Scholar Google

Ortiz Serrano, Salvador (salvador.ortiz@uam.es). Profesor Asociado del Departamento de Economía Aplicada. Facultad de Ciencias Económicas y Empresariales. Universidad Autónoma de Madrid. C/ Francisco Tomás y Valiente, 5. 28049 - Madrid. Buscar otros artículos de este autor en Scholar Google Google 
Jano, M.D. y Ortiz Serrano, S. (2007). Estimación del esfuerzo efectivo para superar la asignatura de estadística descriptiva en las Licenciaturas de Economía y Administración de Empresas.

RELIEVE, v. 13, n. 2, p. 191-202. http://www.uv.es/RELIEVE/v13n2/RELIEVEv13n2_3.htm

\title{
ARTICLE RECORD / FICHA DEL ARTÍCULO
}

\begin{tabular}{|c|c|}
\hline $\begin{array}{l}\text { Reference / } \\
\text { Referencia }\end{array}$ & $\begin{array}{l}\text { Jano, María Dolores y Ortiz Serrano, Salvador (2007). Estimación del esfuerzo efectivo para superar la } \\
\text { asignatura de Estadística Descriptiva en las Licenciaturas de Economía y Administración de Empresa. } \\
\text { RELIEVE, v. 13, n. 2. http://www.uv.es/RELIEVE/v13n2/RELIEVEv13n2_3.htm. Consultado en (po- } \\
\text { ner fecha).. }\end{array}$ \\
\hline Title / Título & $\begin{array}{l}\text { Estimación del esfuerzo efectivo para superar la asignatura de Estadística Descriptiva en las Licenciatu- } \\
\text { ras de Economía y Administración de Empresa. [Student workload estimation to pass a course of statis- } \\
\text { tics in the Degrees of Economy and Business Administration] }\end{array}$ \\
\hline $\begin{array}{l}\text { Authors / } \\
\text { Autores }\end{array}$ & Jano, María Dolores y Ortiz Serrano, Salvador \\
\hline $\begin{array}{l}\text { Review / Re- } \\
\text { vista }\end{array}$ & Revista ELectrónica de Investigación y EValuación Educativa (RELIEVE), v. 13, n. 2 \\
\hline ISSN & $1134-4032$ \\
\hline $\begin{array}{l}\text { Publication } \\
\text { date / } \\
\text { Fecha de } \\
\text { publicación }\end{array}$ & $\begin{array}{l}2007 \text { (Reception Date: } 2006 \text { July 24; Approval Date: } 2007 \text { July 12; Publication Date: } 2007 \text { Septem- } \\
\text { ber 17) }\end{array}$ \\
\hline $\begin{array}{l}\text { Abstract / } \\
\text { Resumen }\end{array}$ & $\begin{array}{l}\text { The convergence process in Higher Education in Europe implies a radical change in the teacher's } \\
\text { practice. One of the fundamental questions in the harmonization process stems from the way credits are } \\
\text { allocated by the ECTS (European Credit Transfer System). This article analyzes diverse methodologies } \\
\text { for the estimation of student workload, with results regarding the time students need to successfully } \\
\text { complete the Descriptive Statistics course in Economics and Business Administration. The obtained } \\
\text { estimated time is slightly below the 25-30 hours of credit that are usually established as a reference. } \\
\text { El proceso de convergencia en la Educación Superior en Europa implica un cambio radical en la ense- } \\
\text { ñanza superior. Una de las cuestiones fundamentes de la armonización radica en el proceso de asigna- } \\
\text { ción de créditos ECTS (European Credit Transfer System). En este artículo se analizan diversas meto- } \\
\text { dologías para la estimación de la carga de trabajo del estudiante y se presentan algunos resultados en } \\
\text { relación al tiempo de estudio para superar la asignatura de Estadística Descriptiva en las licenciaturas de } \\
\text { Economía y ADE. La estimación obtenida está ligeramente por debajo de las 25-30 horas por crédito } \\
\text { que usualmente se establecen como referencia. }\end{array}$ \\
\hline $\begin{array}{l}\text { Keywords / } \\
\text { Descriptores }\end{array}$ & $\begin{array}{l}\text { Data collection, Data Analysis, Active learning, ECTS, Workload } \\
\text { Recogida y análisis de datos, aprendizaje activo, ECTS, Esfuerzo Efectivo del estudiante. }\end{array}$ \\
\hline $\begin{array}{l}\text { Institution / } \\
\text { Institución }\end{array}$ & Universidad Autónoma de Madrid (España). \\
\hline $\begin{array}{l}\text { Publication } \\
\text { site / } \\
\text { Dirección }\end{array}$ & http://www.uv.es/RELIEVE \\
\hline $\begin{array}{l}\text { Language / } \\
\text { Idioma }\end{array}$ & Spanish (Title, abstract and keywords in English ) \\
\hline
\end{tabular}

\section{Revista ELectrónica de Investigación y $\mathbf{E V}$ aluación Educativa (RELIEVE)}

\begin{abstract}
[ ISSN: 1134-4032 ]
(C) Copyright, RELIEVE. Reproduction and distribution of this articles it is authorized if the content is no modified and their origin is indicated (RELIEVE Journal, volume, number and electronic address of the document).

(C) Copyright, RELIEVE. Se autoriza la reproducción y distribución de este artículo siempre que no se modifique el contenido y se indique su origen (RELIEVE, volumen, número y dirección electrónica del documento).
\end{abstract}

